\title{
Toward the True Second Law \\ Part II: The Untenable Assumption of Current Second Law Thermodynamics and the Come into being of the Negentropic Formulation
}

\author{
José C. Íñiguez ${ }^{1}$ \\ ${ }^{1}$ Retired instructor of science and mathematics, $122721^{\text {st }}$ Street, Douglas AZ 85607, USA \\ Correspondence: José C. Íñiguez, Retired instructor of science and mathematics, $122721^{\text {st }}$ Street, Douglas AZ \\ 85607, USA. Tel: 1-520-368-2345. E-mail: iniguez.jose@gmail.com
}

Received: July 30, 2014 Accepted: August 7, 2015 Online Published: August 12, 2015

doi:10.5539/apr.v7n5p39 URL: http://dx.doi.org/10.5539/apr.v7n5p39

\begin{abstract}
Second law thermodynamics', as currently understood, is here proved to be a body of knowledge essentially different from that of Clausius. The rejection on logical grounds of its basic tenet -heat and work as energy forms of the same quality in reversible processes- leads, through a revision of Clausius' work, to a new body of knowledge, 'the Negentropic Formulation', taken by this author to be the true second law of thermodynamics. In it the total entropy change for any given work-producing thermodynamic process is found to be determined by the combination of the opposite sign contributions of the entropic (work degrading) and negentropic (work producing) transformations in it taking place. The efficiency-dependent prevalence of one of these opposites over the other opens the door for irreversible processes with positive, negative, or zero total entropy changes. This notion is at the center of a testable prediction pertaining self-organizing phenomena.
\end{abstract}

Keywords: Clausius' thermodynamics, the second law, the transformation of heat into work, the negentropic formulation, entropy, negentropy

\section{Current Second Law Thermodynamics' Finds Origin in the Correction of Clausius' Work}

\subsection{Introduction}

Most students and studious of thermodynamics believe that second law thermodynamics' as it is known, written about, and taught nowadays is essentially Clausius' work. Small changes here and there, most likely; a lot extended, for certain; but all in all the thermodynamics Clausius constructed by correcting and extending Carnot's work on heat engines and the motive power of heat. This belief, however, bears no correspondence with reality. The version of second law thermodynamics' that the present author and you the reader has been exposed to is actually a radically different version from the body of knowledge constituting Clausius' work on this matter.

This body of knowledge -which will be here referred to as current second law thermodynamics' or CT- must have emerged from an effort to correct the logical shortcomings of Clausius' work; those exposed in the previous paper of this two part series. It seems reasonable to think that when these flaws became known, the law of increasing entropy, apart from becoming the backbone -the essential notion- of second law thermodynamics, as well as an important concept in philosophy and economics, among other parcels of knowledge, had already acquired the status of the 'supreme law of nature' (Eddington, 1929, p.74), and as such taken to be beyond doubt and criticism. In this dogmatic atmosphere it is not unreasonable to think that the corrections of the said flaws were conducted in a way such that the rule of the law of increasing entropy remained supreme, undisputed. As to the 'who' and the 'when' of these corrections, the present author has no factual knowledge. What is indeed factual, as will be proved below, is that the achievement of this objective demanded the replacement of the non-zero values for the two transformations taking place in a reversible cyclical process -notions upon which Clausius constructed his version of the second law of thermodynamics- for values of magnitude equal to zero. The unexplained, surreptitious, and secretive nature of the replacement puts it, in this author's opinion, in a par with an act of magic, or, if the oxymoron is allowed, of magical science. It appears that no effort was made to introduce the necessary corrections into Clausius' work in order to bring to fruition the goal he unsuccessfully 
pursued: that of bringing forward a law which reflected nature's behavior in regard to heat-work inter-conversions, even if the results were in opposition to the putative supreme law of nature. In arbitrarily modifying Clausius' values to retain or guarantee the validity of the law of increasing entropy what we got in return was a distorted image of reality, or to be more precise, an image that only under certain conditions corresponds to that of nature; or even perhaps a construction to suit the purposes of an ideology, not an implausible possibility when due consideration is given to the fact that, as the following quote from Bazarov illustrates, the law of increasing entropy had become a sort of final argument for or against conflicting philosophical positions (See note 1):

The (second) law has caught the attention of poets and philosophers and has been called the greatest achievement of the nineteenth century. Engels disliked it, for it supported opposition to Dialectical Materialism, while Pope Pius XII regarded it as proving the existence of a higher being." (Bazarov, 1964)

The restoration of self-consistency and order produced by these changes was, however, only apparent, as they were achieved via the replacement of one flaw for another. That this is indeed the case will be here proved by showing that the angular stone of current second law thermodynamics' is the absurd notion asserting that in certain situations heat and work are, and in some others are not, energy forms of different quality. These matters are dealt with in Sections 3.2 and 3.3 below.

The numbering of sections, equations, figures and tables here used continues from those of part I.

\subsection{Current Second Law Thermodynamics' Emerges from the Correction of Clausius' Work}

The unveiling of the essential difference existing between Clausius' formulation and $C T$ starts by taking another look at that simple process known as the isothermal and reversible expansion of an ideal gas. This process, corresponding for the purposes of this discussion to process $A E$ in Figure 2(b) and represented in detail in Figure 3 of Part I, is, it should be remembered, the one responsible for bringing forward the work output of the cycle in the form of transformation $\left[Q\left(T_{h}\right) \rightarrow W\right]_{r e v}$. It is to the determination of the entropy change assigned by $C T$ to this transformation that the following argument is directed to.

Irrespective of what perspective -Clausius' or $C T$ - is adopted to study the isothermal and reversible expansion, one fact remains true, before any amount of work $d W$ can be delivered to the mechanical reservoir, the equivalent amount of heat $d Q$ has to flow from the heat reservoir to the gas. This notion leads, as discussed in Section 2.9, to the realization that two transformations are taking place in such a process: the transformation of heat between two bodies - the reservoir and the gas- of essentially the same temperature, and the transformation of this heat into work by the opposed expansion of the gas. In reference to the finite amounts of heat and work $Q$ and $W$ the previous notions lead to the following expression for the total entropy change of reversible process $A E$ :

$$
\Delta S_{C T}[A E]_{r e v}=\Delta S_{C T}\left[Q\left(T_{h}\right) \rightarrow Q\left(T_{h}\right)\right]_{r e v}+\Delta S_{C T}\left[Q\left(T_{h}\right) \rightarrow W\right]_{r e v}
$$

In the previous equation the sub-index $C T$ stands for current second law thermodynamics'. For easy comparison with the results of part I we have designated the process under consideration as $A E$, which is the notation corresponding to Figure 2(b).

The fact that the entropy change associated to the transfer of heat between two bodies of essentially the same temperature is -also with independence of the perspective adopted- equal to zero, allows writing Equation (38) as follows

$$
\Delta S_{C T}[A E]_{\text {rev }}=\Delta S_{C T}\left[Q\left(T_{h}\right) \rightarrow W\right]_{\text {rev }}
$$

The substitution in Equation (39) of the zero total entropy change assigned by $C T$ to an isothermal and reversible expansion, or to any other reversible process for that matter (Pitzer \& Brewer, 1961, p. 83), i.e.

$$
\Delta S_{C T}[A E]_{\text {rev }}=0
$$

leads us to the identification of the entropy change assigned by $C T$ to transformation $\left[Q\left(T_{h}\right) \rightarrow W\right]_{\text {rev }}$ :

$$
\Delta S_{C T}\left[Q\left(T_{h}\right) \rightarrow W\right]_{\text {rev }}=0
$$

The total entropy change for isothermal and reversible compression $E A$, the inverse of the one just discussed, can now be written as follows

$$
\Delta S_{C T}[E A]_{r e v}=\Delta S_{C T}\left[Q\left(T_{h}\right) \rightarrow Q\left(T_{h}\right)\right]_{r e v}+\Delta S_{C T}\left[W \rightarrow Q\left(T_{h}\right)\right]_{r e v}
$$

The same argument leading from Equation (38) to (39) can be used to transit from Equation (42) to the following expression for the total entropy change of process $E A$ :

$$
\Delta S_{C T}[E A]_{r e v}=\Delta S_{C T}\left[W \rightarrow Q\left(T_{h}\right)\right]_{r e v}
$$


Invoking again the zero total entropy change assigned by current second law thermodynamics' to any reversible process $\left(\Delta S_{C T}[E A]_{r e v}=0\right)$ produces the following entropy change for $\left[W \rightarrow Q\left(T_{h}\right)\right]_{r e v}$ :

$$
\Delta S_{C T}\left[W \rightarrow Q\left(T_{h}\right)\right]_{r e v}=0
$$

Armed with this knowledge we can now proceed to evaluate the entropy change for the reversible transformation of heat as it occurs in a reversible heat engine. As noted in Section 2.4 of Part I, this transfer of heat is the sole product of the concatenation of processes $E B-B C-C D-D A$. A representation of this concatenation is given in Figure 6, below. If so, the following expression can be written

$$
\Delta S_{C T}\left[Q_{c}\left(T_{h}\right) \rightarrow Q_{c}\left(T_{c}\right)\right]_{r e v}=\Delta S_{C T}[E B]_{r e v}+\Delta S_{C T}[B C]_{r e v}+\Delta S_{C T}[C D]_{r e v}+\Delta S_{C T}[D A]_{r e v}
$$

The already noted isentropic nature of reversible processes $B C$ and $D A$ can be expressed in the following manner

$$
\Delta S[B C]_{\text {rev }}+\Delta S[D A]_{\text {rev }}=0
$$

The combination of Equations (45) and (46) leads to

$$
\Delta S_{C T}\left[Q_{c}\left(T_{h}\right) \rightarrow Q_{c}\left(T_{c}\right)\right]_{r e v}=\Delta S_{C T}[E B]_{r e v}+\Delta S_{C T}[C D]_{r e v}
$$

The fact that isothermal and reversible processes $E B$ and $C D$ are, respectively, of the same nature as processes $A E$ and $E A$, allows us to write their respective total entropy changes after Equations (39) and (43) as follows:

$$
\Delta S_{C T}\left[Q_{c}\left(T_{h}\right) \rightarrow Q_{c}\left(T_{c}\right)\right]_{r e v}=\Delta S_{C T}\left[Q_{c}\left(T_{h}\right) \rightarrow W_{c}\right]_{r e v}+\Delta S_{C T}\left[W_{c} \rightarrow Q_{c}\left(T_{c}\right)\right]_{r e v}
$$

The fact made evident by Equations (41) and (44) that for current second law thermodynamics' the reversible transformations of heat into work and vice-versa take place with a zero entropy change leads us, finally, to the following total entropy change for the concatenation of processes $E B-B C-C D-D A$ or equivalently, for transformation $\left[Q_{c}\left(T_{h}\right) \rightarrow Q_{c}\left(T_{c}\right)\right]_{\text {rev }}$, i.e.,

$$
\Delta S_{C T}\left[Q_{c}\left(T_{h}\right) \rightarrow Q_{c}\left(T_{c}\right)\right]_{r e v}=0
$$

For its inverse we can write

$$
\Delta S_{C T}\left[Q_{c}\left(T_{c}\right) \rightarrow Q_{c}\left(T_{h}\right)\right]_{\text {rev }}=0
$$

We can now proceed to calculate the total or universe entropy change for a reversible heat engine in terms of the combined total entropy changes for processes $A E$ and $E B-B C-C D-D A$, or equivalently, as allowed by Equations (39) and (45), in terms of the combined entropy changes of the two transformations brought forward by those processes, as follows

$$
\Delta S_{C T}[C y c l e]_{r e v}=\Delta S_{C T}\left[Q\left(T_{h}\right) \rightarrow W\right]_{r e v}+\Delta S\left[Q_{c}\left(T_{h}\right) \rightarrow Q_{c}\left(T_{c}\right)\right]_{r e v}=0+0=0
$$

Note that the same total entropy change for a reversible heat engine is provided by both, Clausius' construction and current second law thermodynamics'. The path leading to this result is, however, radically different in one and the other. Clausius produces a zero, as Equation (29) makes evident, via the addition of two non-zero quantities. Current thermodynamics does it, on its part -as the previous equation shows- via the addition of two zeros.

In order to be able to fully contrast Clausius formulation with $C T$ we must develop for the latter a table similar to Table 1. In order to do this we must ascertain the values $C T$ ascribes to the following transformations: $\left[Q_{c}\left(T_{h}\right) \rightarrow Q_{c}\left(T_{c}\right)\right]_{i r r}$ and $[W \rightarrow Q(T)]_{i r r}$. The former can be obtained via the application of Equation (13) to the heat reservoirs taking part in this irreversible heat transfer process. When this is done we get:

$$
\Delta S_{C T}\left[Q_{c}\left(T_{h}\right) \rightarrow Q_{c}\left(T_{c}\right)\right]_{i r r}=-\frac{Q_{c}}{T_{h}}+\frac{Q_{c}}{T_{c}}
$$

As for $[W \rightarrow Q(T)]_{i r r}$ all we have to do is to carry over from noted $C T$ textbook author's Pitzer and Brewer (1961, pp. 85-86) and Schmidt (1966, p. 130) the value by them ascribed to the irreversible degradation of work into heat:

$$
\Delta S_{C T}[W \rightarrow Q(T)]_{i r r}=Q / T
$$

evident in their following respective quotes:

"If an amount of work $\delta W$ is degraded to heat of temperature $T$, the increase in entropy is $d S_{i r r}=\delta W / T$."

"In the course of the process (one cycle of a heat engine) the quantity of work W...is stored in the form of the potential energy of the weight.

If we allow the weight to sink back to its original level and if its energy is simultaneously transformed into heat through friction, the heat of friction $Q_{f}=W$ being added to the source $T_{o}$ (the cold reservoir), the entropy of the latter will increase by $\Delta S=Q_{f} / T_{o}=W / T_{o}$. 
These last two values, it should be noted, are identical to those of Clausius' thermodynamics.

The entropy changes for what can be called the fundamental or essential transformations characterizing Clausius' formulation haven been annotated in Table 2 vis-à-vis those of current second law thermodynamics'. The obvious difference existing between these two sets evince our previous assertion that Clausius' second law thermodynamics and current second law thermodynamics' are essentially different from one another. The values for the two transformations taking place in a reversible heat engine, those upon which Clausius built his second law, are found in current second law thermodynamics' inexplicably transiting from $-Q / T_{h}$ and $\left(-Q_{c} / T_{h}\right)+\left(Q_{c} / T_{c}\right)$, to a value of zero.

Table 2. Clausius' set of values for the transformations are here contrasted with those sanctioned by current thermodynamics. Note that in the latter while all reversible transformations take place with zero entropy changes, all those of an irreversible nature convey positive entropy changes

\begin{tabular}{lll}
\hline Transformation & Clausius' work & $C T$ \\
\hline$\left[Q\left(T_{h}\right) \rightarrow W\right]_{\text {rev }}$ & $-Q / T_{h}$ & 0 \\
{$\left[W \rightarrow Q\left(T_{h}\right)\right]_{\text {rev }}$} & $Q / T_{h}$ & 0 \\
{$\left[W \rightarrow Q\left(T_{h}\right)\right]_{\text {irr }}$} & $Q / T_{h}$ & $Q / T_{h}$ \\
{$\left[Q_{c}\left(T_{h}\right) \rightarrow Q_{c}\left(T_{c}\right)\right]_{\text {rev }}$} & $\left(-Q_{c} / T_{h}\right)+\left(Q_{c} / T_{c}\right)$ & 0 \\
{$\left[Q_{c}\left(T_{c}\right) \rightarrow Q_{c}\left(T_{h}\right)\right]_{\text {rev }}$} & $\left(-Q_{c} / T_{c}\right)+\left(Q_{c} / T_{h}\right)$ & 0 \\
{$\left[Q_{c}\left(T_{h}\right) \rightarrow Q_{c}\left(T_{c}\right)\right]_{i r r}$} & $\left(-Q_{c} / T_{h}\right)+\left(Q_{c} / T_{c}\right)$ & $\left(-Q_{c} / T_{h}\right)+\left(Q_{c} / T_{c}\right)$ \\
\hline
\end{tabular}

It is impossible to miss from Table 2 the fact that current second law thermodynamics' assigns a zero total entropy change to all reversible transformations and a positive one to those irreversible. In assuring a zero total entropy change for any reversible process and a positive total entropy change for those irreversible, this set of values makes it impossible to fault the law of increasing entropy, and in the process safeguards from any doubt -well, almost any doubt- the entropic evolution of the universe and its eventual demise in the form of its heat death.

The evidence to be provided in what follows will unveil the fact that the 'success' of $C T$ in providing a foolproof body of knowledge was only apparent, as the process of getting there involved, as already mentioned, a trade of flaws. Here we find the thermodynamic version of that old tale of digging one hole to cover another. It is to the identification of the new logical hole that the following argument is directed to.

\section{The Untenable Position of Current Second Law Thermodynamics}

To understand why the assignment of a zero entropy change to the reversible transformation of heat into work is unacceptable, let us consider the processes depicted in Figure 5.

In it process (a) represents the reversible transfer of an amount of heat $d Q$ between two bodies (heat reservoirs) of essentially the same temperature $T$. Process (b) represents, on its part, an isothermal and reversible ideal gas expansion in which, concomitant to the transfer of the amount of heat $d Q$ between two bodies -the heat reservoir and the gas- of essentially the same temperature, we find the production of an equivalent amount of work $d W$ by the opposed expansion of the gas. The essential fact that needs here to be considered is that according to current thermodynamic wisdom both of these processes are, entropy-wise, indistinguishable of one another as both of them take place with a zero total entropy change. On this perspective energy upgrading process (b), through which the disorganized energy form we call heat becomes the ordered energy we call work, is indistinguishable from a heat transfer process. For the second law of thermodynamics, as currently understood, work and heat are -in reversible processes- energy forms of the same quality. The constructive, organizing, and transforming powers subsumed by 'work' evident in all the structures around us, in the change in condition it can produce in other bodies, in its capability of restoring spent gradients and make spontaneous the non-spontaneous etc., are under this perspective erased, neglected, ignored. What besides heating a colder body and cooling a hotter one can be achieved by a heat transfer process such as (a)? Nothing! The fact that none of the previous tasks can be achieved with heat itself i.e., without it being first converted into work, should be reason enough to reject the notion of heat and work being entropically indistinguishable in reversible processes. Those thermodynamicists denying a quality gain or entropy change in the -necessarily reversible- transformation of heat into work might think differently if once their homes were deprived of electricity, they had to rely on fire as energy source. In a situation like this they are most likely to agree that their 'quality' of life has radically decreased. 


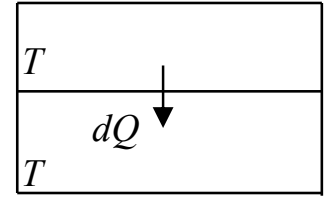

(a)

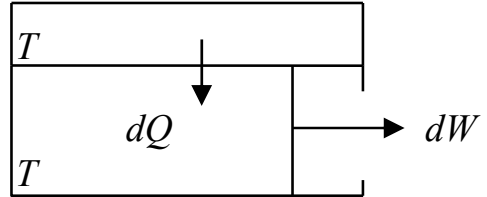

(b)

Figure 5. Process (a) represents the transfer of an amount of heat $d Q$ between two bodies of essentially the same temperature. Here no work is outputted. Process (b) represents, on its part, the isothermal and reversible expansion of an ideal gas where an amount of heat $d Q$ is also transferred between two bodies - the heat reservoir and the ideal

gas- at essentially the same temperature. The difference being that here the expansion of the gas manages to

transform $d Q$ into an equivalent amount of work $d W$ that appears in an appropriate mechanical reservoir. For current second law thermodynamics these two processes are entropically indistinguishable as they both take place with a zero total entropy change. This position makes heat and work energy forms of the same quality

In making the whole new set of capabilities gained in the transformation of heat into work taking place in process (b) indistinguishable from the practically non-existent capability of the effects- barren heat transfer represented in (a), current thermodynamics is nothing short of equating equilibrium -characterized by its inability for work output- with evolution, change, or transformation -the sine qua non condition for work production; or equivalently, of equating rest with motion. In this last perspective processes (a) and (b) re-edit the confrontation between the philosophical positions of Parmenides and Heraclitus. When confronted with it the 'correctors' of Clausius' work responsible for bringing forward $C T$ wasted no time in finding an efficient solution: They simply, in a way of speaking, 'killed' Heraclitus!

As the following statements attest, the previous considerations are in line with the intuitive, commonsensical lore existing around the different quality of these two energy forms:

"Entropy is a measure for the quality of the energy in the system." (Jantsch, 1983, p. 25);

"In practical terms what a steam engine does is to convert heat into work, with work simply meaning a more useful and organized form of energy." (Coveney \& Highfield, 1990, p. 149);

"But what about change of form of motion, or so-called energy? If we change heat into mechanical motion or vice versa, is not the quality altered while the quantity remains the same? (Engels, 1964, p. 64);

"...there can be no escape from the conclusion that there is an intrinsic difference between heat and work." (Smith \& Van Ness, 1965, p. 175);

"The entropy change of the system plus surroundings may be viewed as a quantitative measure, or index, of the degradation of energy as work to energy as heat, as a consequence of irreversible elements in the process under study." (Weber \& Meissner, 1957, p. 168).

In recognizing an intrinsic difference in quality between heat and work, the previous notions appear to contradict current second law thermodynamics' position of making reversible heat/work inter-conversions constant entropy processes. For if indeed heat and work are energy forms of different quality then any transformation of one into the other has to be accompanied by a measure -a non-zero entropy change- of the quality gained or lost in it. Beyond determining whether the change of quality is permanent or not, the nature of the path (reversible or irreversible) has no other bearing in this matter; certainly not the power of making the difference in quality between heat and work appear under certain conditions, such as the irreversible transformation of work into heat, and disappear in others, such as the reversible heat-to-work inter-conversions. As Clausius originally stated, "...the value of a change from work into heat must be proportional to the amount of heat generated and...beyond this it can only depend on the temperature." (Clausius, p. 98). If so, the reversible transformation of heat into work must reflect the gain in quality with -in accord to Clausius' convention on this matter- an entropy decrease, while both, the reversible and irreversible transformations of work into heat must convey positive entropy changes as a reflection of the -transitory in the former and permanent in the latter- loss of quality involved.

Our agreement with Clausius in that the reversible inter-conversions between heat and work convey entropy changes of magnitudes 


$$
\Delta S\left[Q\left(T_{h}\right) \rightarrow W\right]_{r e v}=-Q / T_{h}
$$

and

$$
\Delta S\left[W \rightarrow Q\left(T_{h}\right)\right]_{\text {rev }}=Q / T_{h}
$$

implies necessarily our rejection of current second law thermodynamics'. Even so, Equations (54) and (55) are as far as our agreement with Clausius' work actually goes. The reason behind this statement will become evident in the following sections. Let us then make clear that given our rejection of $C T$ what we propose to do is go back to Clausius' work and guided - in addition to the notions represented by the last two equations- by the light of reason and constrained by logical soundness -but certainly not by preconceived notions or religious or philosophical bias- subject Clausius work to a profound review. The body of knowledge to emerge from such an undertaking is what constitutes the Negentropic Formulation $(N F)$, in this author's opinion the True Second Law of Thermodynamics. Let us then go back to Clausius' work in order to recognize his only logical flaw, the one from which all the inconsistencies unveiled in Part I originate.

\section{The Negentropic Formulation}

\subsection{Clausius' Conceptual Error or the True Role of Isentropic Processes in Carnot's Cycle}

Let us center our attention on the concatenation of processes $E B-B C-C D-D A$ depicted in Figure 6(a), below. As should be remembered it is this concatenation the one responsible for producing in a heat engine the reversible transfer of heat from the hot to the cold reservoir. As known, in isothermal and reversible expansion $E B$ an amount of heat $Q_{c}$ of temperature $T_{h}$ transferred from the hot reservoir to the ideal gas is transformed into an equivalent amount of work $W_{c}$. In doing so process $E B$ brings forward transformation $\left[Q_{c}\left(T_{h}\right) \rightarrow W_{c}\right]_{\text {rev }}$ with an associated entropy change, in agreement with Equation (54), of $-Q_{c} / T_{h}$. If we assume a value of zero for the entropy of the universe at point $E$ then its entropy at point $B$ is equal to $S_{B}=-Q_{c} / T_{h}$. Let us now recognize that process $B C$ not only changes the volume and temperature of the gas from its original values at $B$ to those ruling at $C$; it also changes the heat reservoir in contact with the gas from that of temperature $T_{h}$ to that of temperature $T_{c}$. The mechanical reservoir and the work it safe-keeps form now part of the new situation. The fact that the condition of all these bodies defines at any moment the condition of the universe allows us to understand that what process $B C$ actually accomplishes is the substitution of universe $B$ with universe $C$, and in doing so brings forward an entropy change consisting in switching the negentropy $\left(-Q_{c} / T_{h}\right)$ associated to the production of $W_{c}$ out of heat of temperature $T_{h}$ for that which in the amount of $-Q_{c} / T_{c}$ would have arisen had $W_{c}$ been generated from heat of temperature $T_{c}$. The rationale is actually very simple. Once $W_{c}$ and the rest of the universe arrive at $C$, this work becomes indistinguishable from a similar amount generated out of heat of temperature $T_{c}$. In the universe exchange produced by process $B C, W_{c}$ is in a manner of speaking 'decoupled' from heat of temperature $T_{h}$ and 'coupled' to heat of temperature $T_{c}$. The palpable effect of this decoupling /coupling is the correction from one temperature to another of the negentropy associated to $W_{c}$. In doing this, adiabatic and reversible process $B C$ brings forwards what in rigor is the transformation of a transformation, i.e. $\left\{\left[Q_{c}\left(T_{h}\right) \rightarrow W_{c}\right] \rightarrow\left[Q_{c}\left(T_{c}\right) \rightarrow W_{c}\right]\right\}$, with an associated entropy change (final entropy minus initial entropy) of $\left(-Q_{c} / T_{c}\right)-\left(-Q_{c} / T_{h}\right)$ or equivalently, of $\left(-W_{c} / T_{c}\right)-\left(-W_{c} / T_{h}\right)$. The decoupling / coupling referred can also take place, as will be shown below, with work remaining once an isothermal and reversible compression has taken place. For economy reasons this transformation will be from now on designated as the $W$-Transformation and represented, in general, as $\left[W\left(T_{1}\right) \rightarrow W\left(T_{2}\right)\right]$ or as $[W]_{B C}$. With this notation we can write

$$
\Delta S\left[W_{c}\left(T_{h}\right) \rightarrow W_{c}\left(T_{c}\right)\right]=\Delta S\left[W_{c}\right]_{B C}=\left(-Q_{c} / T_{c}\right)-\left(-Q_{c} / T_{h}\right)
$$

With these antecedents in place we can write the entropy of the universe at $C$ as follows

$$
S_{c}=S_{B}+\Delta S[W]_{B C}+\Delta S[B C]
$$

The previously given entropy values for $S_{B}$ and $\left[W_{c}\right]_{B C}$ in combination with the fact that process $B C$ is isentropic, allows writing the previous equation as follows:

$$
S_{c}=\left(-Q_{c} / T_{h}\right)+\left[\left(-Q_{c} / T_{c}\right)-\left(-Q_{c} / T_{h}\right)\right]+0=-Q_{c} / T_{c}
$$

The entropy change associated to what we have called the W-Transformation was unrecognized by Clausius. In his analysis processes $B C$ and $D A$ are simple connectors between the hot and cold isotherms. Had he been aware of it, the science of thermodynamics would have taken its true form from the very beginning. The effect in question was also unrecognized by the 'correctors' of Clausius work. Its oversight had, however, no consequence as the W-Transformation in this case would be switching a zero for a zero, as for $C T$ the entropy change for the transformation of heat into work is zero at any temperature. 


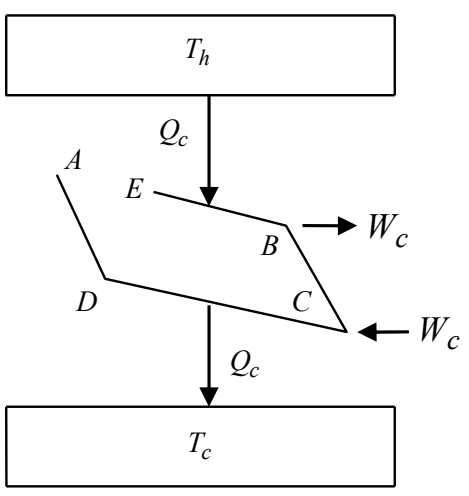

(a)

$\Delta S[E B]=-Q_{c} / T_{h}$

$\Delta S[B C]=\left(-Q_{c} / T_{c}\right)+\left(Q_{c} / T_{h}\right)$

$\triangle S[C D]=Q_{c} / T_{c}$

$\triangle S[D A]=0$

$\triangle S[E B C D A]=0$

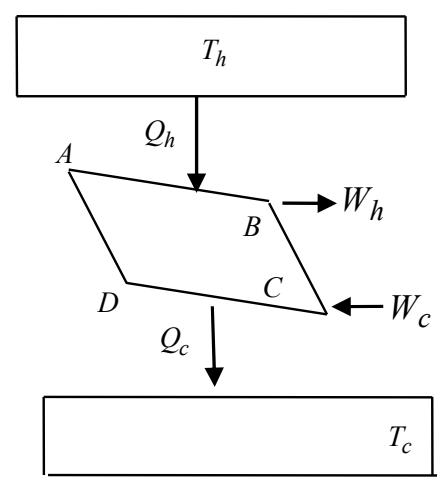

(b)

$$
\begin{aligned}
& \Delta S[A B]=-Q_{h} / T_{h} \\
& \Delta S[B C]=\left(-Q_{h} / T_{c}\right)+\left(Q_{h} / T_{h}\right) \\
& \Delta S[C D]=Q_{c} / T_{c} \\
& \Delta S[D A]=\left(-Q / T_{h}\right)+\left(Q / T_{c}\right) \\
& \Delta S[A B C D A]=-Q / T_{h}
\end{aligned}
$$

Figure 6. Figure (a) represents the concatenation of processes required to produce in a heat engine the reversible transformation of heat from one temperature to another. If traveled in the sequence $E B C D A$ the heat will be reversibly transformed from the hot to the cold reservoir. If instead the path $A D C B E$ is followed, heat will be transferred in the opposite direction. Figure (b) represents, on its part, a reversible cyclical process. The entropy changes written below these figures are the one calculated according to the Negentropic Formulation with the concourse of its associated concept: The W-Transformation

Continuing with the concatenation of processes being considered, let us agree that the universe finds itself now at $C$ with an entropy, as shown by Equation (58), of $-Q_{c} / T_{c}$. The next process of the concatenation is isothermal and reversible compression $C D$. Here the work $W_{c}$ available in the mechanical reservoir is expended along this process and the spent work released as heat at the temperature of the cold reservoir. In doing so process $C D$ brings forward transformation $\left[W_{c} \rightarrow Q_{c}\left(T_{c}\right)\right]_{r e v}$ with an associated entropy change of $Q_{c} / T_{c}$. The entropy of the universe at $D$ can now be calculated as the summation of the entropy at $C$ plus the entropy change of process $C D$. When this is done we get: $S_{D}=\left(-Q_{c} / T_{c}\right)+\left(Q_{c} / T_{c}\right)=0$. This is a general result of the Negentropic Formulation: For any two isothermal and reversible ideal gas expansions $E B\left(T_{1}\right)$ and $D C\left(T_{2}\right)$ for which $W_{E B}=W_{D C}$ (same amount of work produced by one and the other) and $T_{1} V_{B}^{\gamma-1}=T_{2} V_{C}^{\gamma-1}$ (points $B$ and $C$ lying on the same reversible adiabat), it is true that $S_{E}=S_{D}$ (The entropy of the universe at $B$ and $D$ are identical).

Let us now agree that at point $D$ no more work is available in the universe. Process $D A$, the last leg of the concatenation, has thus no work to decouple / couple and consequently no negentropy to correct from one temperature to another. Here process $D A$ assumes the role of simple connector between the hot and cold isotherm. If so:

Therefore

$$
\Delta S[0]_{D A}=\left[\left(-0 / T_{h}\right)-\left(-0 / T_{c}\right)\right]=0
$$

$$
S_{A}=S_{D}+\Delta S[0]_{D A}+\Delta S[D A]=0
$$

We are now in possession of all the elements required to calculate the entropy change assigned by the Negentropic Formulation ( $N F$ ) to concatenation $E B-B C-C D-D A$, or equivalently, to the reversible transformation of $Q_{c}$ from $T_{h}$ to $T_{c}$. Recognition of the fact that the said concatenation is defined by the transit of the universe from condition $E$, with $S_{E}=0$, to condition $A$, with $S_{A}=0$, allows us to write $\Delta S_{N F}\left[Q_{c}\left(T_{h}\right) \rightarrow Q_{c}\left(T_{c}\right)\right]_{r e v}=S_{A}-S_{E}=0$. An alternative path to this result can be obtained by writing

$$
\Delta S_{N F}\left[Q_{c}\left(T_{h}\right) \rightarrow Q_{c}\left(T_{c}\right)\right]_{r e v}=\Delta S\left[Q_{c}\left(T_{h}\right) \rightarrow W_{c}\right]_{r e v}+\Delta S\left[W_{c}\right]_{B C}+\Delta S\left[W_{c} \rightarrow Q_{c}\left(T_{c}\right)\right]+\Delta S[0]_{D A}
$$

On substitution of the corresponding values for the entropy changes of the transformations there involved, the previous equation becomes

$$
\Delta S_{N F}\left[Q_{c}\left(T_{h}\right) \rightarrow Q_{c}(T)\right]_{r e v}=\left(-Q_{c} / T_{h}\right)+\left[\left(-Q_{c} / T_{c}\right)-\left(-Q_{c} / T_{h}\right)\right]+\left(Q_{c} / T_{c}\right)+0
$$


On performance of the indicated operations this equation reduces to

$$
\Delta S_{N F}\left[Q_{c}\left(T_{h}\right) \rightarrow Q_{c}\left(T_{c}\right)\right]_{r e v}=0
$$

If so, then

$$
\Delta S_{N F}\left[Q_{c}\left(T_{c}\right) \rightarrow Q_{c}\left(T_{h}\right)\right]_{\text {rev }}=0
$$

A comparison between Equations (27) and (61) makes clear the point of separation between Clausius' second law and the Negentropic Formulation. Thus, while in the former the reversible transfer of heat taking place in a reversible cyclical process comes out of the combination $\left[Q_{c}\left(T_{h}\right) \rightarrow W_{c}\right]_{r e v}+\left[W_{c} \rightarrow Q_{c}\left(T_{c}\right)\right]_{\text {rev }}$; in the latter it comes out as the product of the combination of those two same transformations plus the two W-Transformations there operating. This conceptual difference finds expression in the different entropy changes this transformation conveys in one case and the other: positive in the former, zero in the latter. Let us also note here that even if for the wrong reason, $C T$ also assigns a zero entropy change to this transformation. As Equation (48) makes evident, this transformation is in $C T$ rationalized the same way Clausius did. The zero arises here, however, as a consequence of $C T$ making zero the entropy change of heat-work inter-conversions. The right result appears this way at the cost of introducing the notion asserting the quality identity between heat and work. This is what we meant above when we said that $C T$ removed one flaw at the cost of introducing another

In order to fully characterize the Negentropic Formulation and thus be able to contrast it with both Clausius' construction and current second law thermodynamics' we need the values for transformations $\Delta S\left[Q_{c}\left(T_{h}\right) \rightarrow Q_{c}\left(T_{c}\right)\right]_{i r r}$ and $\Delta S\left[W \rightarrow Q\left(T_{c}\right)\right]_{i r r}$. For these we will adopt the values given assigned to them by $C T$ as given in Equations (52) and (53). The reason for this boils down to the fact that the procedure to obtain them it is the same in $C T$ and $N F$. In the former, a direct application of the entropy defining equation to both of the reservoirs involved in such a process. In the latter we simply recognize the soundness of Schmidt's reasoning given in regard to Equation (53).

At this point the fundamental set of values of the Negentropic Formulation is complete. In order to simplify the comparison between the values that these three different formulations of the second law of thermodynamics assign to the reversible and irreversible inter-conversions between heat and work as well as those of heat from one temperature to another, is that Table 3 has been constructed.

With the Negentropic Formulation properly characterized via the set of corresponding values of Table 3, we can address the problem of determining the total or universe entropy change by it associated to a reversible cyclical process. For the same reasons advanced in regard to Equation (51), the total or universe entropy change for the reversible cycle will be written as the summation of the entropy changes for the two transformations there taking place, i.e.

$$
\Delta S_{N F}[\text { Cycle }]_{r e v}=\Delta S\left[Q\left(T_{h}\right) \rightarrow W\right]_{r e v}+\Delta S\left[Q_{c}\left(T_{h}\right) \rightarrow Q_{c}\left(T_{c}\right)\right]_{r e v}
$$

Replacement of the indicated entropy changes for the values annotated in Table 3 produces

$$
\Delta S_{N F}[\text { Cycle }]_{\text {rev }}=-Q / T_{h}
$$

The previous result indicates that contrary to common wisdom, the total entropy change associated to one cycle in the operation of a reversible heat engine is negative, in other words it is a negentropic process; the negentropy created being proportional to the work produced. It needs to be recognized here that at the end of one cycle the only body returning to its initial condition is the ideal gas acting as variable body. The other three bodies there involved: the heat and mechanical reservoirs, are found in a condition different than the one they originally had. These three changes remaining in the universe are (1) the one sustained by the hot reservoir in releasing an amount of heat $Q_{h} ;(2)$ the one sustained by the cold reservoir in receiving an amount of heat $Q_{c}$; and (3) the one sustained by the mechanical reservoir in receiving an amount of work $W$ produced out of heat of temperature $T_{h}$. The entropy changes of the heat reservoirs do not appear on Equation (66) on reason of their magnitudes combining to a value of zero, i.e. $\left(-Q_{h} / T_{h}\right)+\left(Q_{c} / T_{c}\right)=0$. The fact that it is the production of $W$ the only non-compensated change left in the universe explains why the only term appearing in this equation is $-Q / T_{h}$. If to Equation (66) we now add a zero in the form of $\left(-Q_{h} / T_{h}\right)+\left(Q_{c} / T_{c}\right)$ we will be getting what in all rigor is the Negentropic Formulation's expression for the total entropy change of a reversible cycle:

$$
\Delta S_{N F}[\text { Cycle }]_{r e v}=-\frac{Q_{h}}{T_{h}}+\frac{Q_{c}}{T_{c}}-\frac{Q}{T_{h}}
$$

Given the radically new perspective brought about by the $\mathrm{W}$-Transformation, an analysis similar to that leading to Equation (66) will be carried on for the reversible cycle $A B C D A$ depicted in Figure 6(b). Let us start by writing the entropy changes associated to processes $A B,\left[W_{h}\right]_{B C}, C D$, and $[W]_{D A}$, as follows 


$$
\begin{gathered}
\Delta S_{N F}[A B]=\Delta S_{N F}\left[Q_{h}\left(T_{h}\right) \rightarrow W_{h}\right]_{r e v}=-Q_{h} / T_{h} \\
\Delta S_{N F}\left[W_{h}\right]_{B C}=\left(-Q_{h} / T_{c}\right)-\left(-Q_{h} / T_{h}\right) \\
\Delta S_{N F}[C D]_{r e v}=\Delta S_{N F}\left[W_{c} \rightarrow Q_{c}\left(T_{c}\right)\right]_{r e v}=Q_{c} / T_{c} \\
\Delta S[W]_{D A}=\left(-Q / T_{h}\right)-\left(-Q / T_{c}\right)
\end{gathered}
$$

The previous equations reflect the following facts: (1) that in the case being considered process $A B$ transforms an amount of heat $Q_{h}\left(T_{h}\right)$ into an equivalent amount of work $W_{h}$ with an associated entropy change of $-Q_{h} / T_{h}$; (2) The W-Transformation operating along process $B C$ corrects the negentropy carried on by $W_{h}$ as part of the hot isotherm for that it conveys as part of the cold isotherm; (3) The portion $W_{c}$ out of the amount of work $W_{h}$ available in the mechanical reservoir is used to carry on compression $C D$. The spent work ends up as an equivalent amount of heat in the cold reservoir. The entropy change for this process is $Q_{c} / T_{c}$; (4) The amount of work available in the mechanical reservoir at point $D$ is $W=W_{h}-W_{c}$. As part of the cold isotherm, the negentropy associated to $W$ amounts to $-Q / T_{c}$. The W-Transformation operating along $D A$ corrects this value for the one that corresponds to $W$ as part of the hot isotherm.

Table 3. The set of values characterizing the Negentropic Formulation is here shown vis-à-vis those of Clausius' construction and current second law thermodynamics. Note that the difference with Clausius work comes down to the entropy changes of the reversible transformations of heat

\begin{tabular}{llll}
\hline Transformation & Clausius' & $C T$ & $N F$ \\
\hline$\left[Q\left(T_{h}\right) \rightarrow W\right]_{r e v}$ & $-\frac{Q}{T_{h}}$ & 0 & $-\frac{Q}{T_{h}}$ \\
{$\left[W \rightarrow Q\left(T_{h}\right)\right]_{r e v}$} & $\frac{Q}{T_{h}}$ & 0 & $\frac{Q}{T_{h}}$ \\
{$\left[W \rightarrow Q\left(T_{h}\right)\right]_{i r r}$} & $\frac{Q}{T_{h}}$ & $\frac{Q}{T_{h}}$ & $\frac{Q}{T_{h}}$ \\
{$\left[Q_{c}\left(T_{h}\right) \rightarrow Q_{c}\left(T_{c}\right)\right]_{r e v}$} & $Q_{c}\left(\frac{1}{T_{c}}-\frac{1}{T_{h}}\right)$ & 0 & 0 \\
{$\left[Q_{c}\left(T_{c}\right) \rightarrow Q_{c}\left(T_{h}\right)\right]_{r e v}$} & $Q_{c}\left(\frac{1}{T_{h}}-\frac{1}{T_{c}}\right)$ & 0 & 0 \\
{$\left[Q_{c}\left(T_{h}\right) \rightarrow Q_{c}\left(T_{c}\right)\right]_{i r r}$} & $Q_{c}\left(\frac{1}{T_{c}}-\frac{1}{T_{h}}\right)$ & $Q_{c}\left(\frac{1}{T_{c}}-\frac{1}{T_{h}}\right)$ & $Q_{c}\left(\frac{1}{T_{c}}-\frac{1}{T_{h}}\right)$ \\
\hline
\end{tabular}

Since the combination of processes $A B,\left[W_{h}\right]_{B C}, C D$, and $[W]_{D A}$ define the reversible cycle being considered, the summation of their entropy changes must define its total (or universe) entropy change. Therefore

$$
\Delta S_{N F}[\text { Cycle }]_{r e v}=\left(-Q_{h} / T_{h}\right)+\left[\left(-Q_{h} / T_{c}\right)-\left(-Q_{h} / T_{h}\right)\right]+\left(Q_{c} / T_{c}\right)+\left[\left(-Q / T_{h}\right)-\left(-Q / T_{c}\right)\right]
$$

Performance of the indicated operations produces the following result

$$
\Delta S_{N F}[\text { Cycle }]_{\text {rev }}=-Q / T_{h}
$$

The agreement existing between Equations (66) and (73) is a testament of the self-consistency of the Negentropic Formulation. In the next section it will be shown that Equation (67) retains its form when applied to irreversible cyclical processes.

\subsection{The Negentropic Formulation on Irreversible Cyclical Processes}

The problem at hand is to determine in accord with the Negentropic Formulation the total entropy change for a non-reversible cyclical process. Let us then focus our attention on Figure 7. There process (a) represents a cyclical process which working with an efficiency $\eta, \eta\left\langle\eta_{\text {rev }}\right.$, manages to produce an amount of work $W=\eta Q_{h}$ out of an amount of heat $Q_{h}$ received by it from the hot reservoir of temperature $T_{h}$. In the figure, processes (b) and (c) represent the reversible and irreversible components of (a). In process (b), $Q_{h}^{*}$ and $Q_{c}^{*}$ represent, respectively, the amounts of heat coming out of the hot reservoir, and taken in by the cold reservoir had the production of $W$ taken place in a reversible fashion. Here $Q_{h}^{*}-Q_{c}^{*}=Q=W$ and $W=\eta_{\text {rev }} Q_{h}^{*}$. On 
reason of $\eta_{\text {rev }}>\eta$ we have that $\left.W / Q_{h}^{*}\right\rangle W / Q_{h}$ or, equivalently, that $Q_{h}>Q_{h}^{*}$. In accord with Equation (66) we can write the total entropy change for process (b) as:

$$
\Delta S_{r e v}=-W / T_{h}=-Q / T_{h}
$$

That the same work output demands a larger intake of heat in (a) than in (b) can only be explained by the fact that the amount of heat $Q_{i}$ quantifying the difference $Q_{h}-Q_{h}^{*}=Q_{i}$, manages to bypass in (a) the work producing circuit of the variable body. In its irreversible transit this amount of heat carries with it an untapped work producing potential, the lost work, in the amount of

$$
W_{\text {lost }}=Q_{i}\left(T_{h}-T_{c}\right) / T_{h}
$$

If this work producing potential had been harnessed then instead of $W$, process 7(a) would have had a work output of $W+W_{\text {lost }}=W_{\text {rev }}=\eta_{\text {rev }} Q_{h}$.

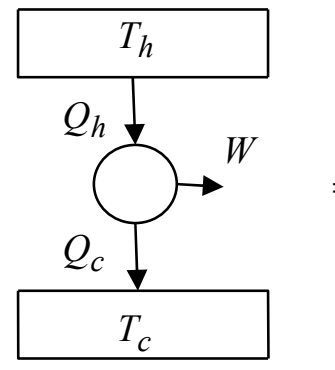

(a)

A simple cyclical process

$\Delta S_{N F}[\text { Cycle }]_{i r r}$

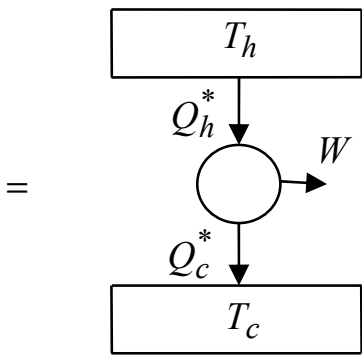

(b)

The reversible component

$\Delta S_{\text {rev }}$

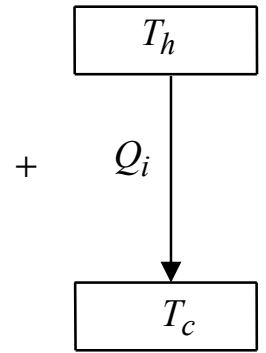

(c)

The irreversible component

$\Delta S_{\text {irr }}$

Figure 7. A simple cyclical process of efficiency $\eta$ is here separated into its reversible and irreversible components. The entropy change for this process is determined by the summation of the contributions of its components

The irreversible component depicted in (c) refers to this amount of heat $Q_{i}$ flowing in a direct, irreversible fashion from the hot to the cold reservoir. In correspondence to its irreversible nature its total entropy change amounts to

$$
\Delta S_{\text {irr }}=Q_{i}\left(T_{h}-T_{c}\right) /\left(T_{h} T_{c}\right)=W_{\text {lost }} / T_{c}
$$

Let us also note that on reason of $Q_{h}-Q_{c}=Q_{h}^{*}-Q_{c}^{*}$, we can write $Q_{h}-Q_{h}^{*}=Q_{c}-Q_{c}^{*}=Q_{i}$. Implicit in this last equation is the fact that reversible is any cycle for which $Q_{i}=0$.

Let us note that combined, processes (b) and (c) reproduce process (a). Thus, $Q_{h}^{*}+Q_{i}=Q_{h}, Q_{c}^{*}+Q_{i}=Q_{c}$, and $W(b)+W(c)=W(a)$. It is on reason of this that we can express the total entropy change of process (a) as the summation of the total entropy changes of its reversible and irreversible components (b) and (c), as

$$
\Delta S_{N F}[\text { Cycle }]_{i r r}=\Delta S_{i r r}+\Delta S_{\text {rev }}
$$

In order to be able to write the final expression for $\Delta S_{N F}[C y c l e]_{i r r}$ we will proceed to perform some required algebraic manipulations on $\Delta S_{i r r}$ as given by Equation (76).

Let us start by writing Equation (76) as follows

$$
\Delta S_{i r r}=-\frac{Q_{i}}{T_{h}}+\frac{Q_{i}}{T_{c}}
$$

The respective replacement of $Q_{i}$ by $Q_{h}-Q_{h}^{*}$ and $Q_{c}-Q_{c}^{*}$ in the first and second terms on the previous equation leads, after rearrangement, to the following expression

$$
\Delta S_{i r r}=\left(-\frac{Q_{h}}{T_{h}}+\frac{Q_{c}}{T_{c}}\right)-\left(-\frac{Q_{h}^{*}}{T_{h}}+\frac{Q_{c}^{*}}{T_{c}}\right)
$$


Recognition of the fact that the last of the right hand side parenthesis is equal to zero reduces Equation (79) to the following form.

$$
\Delta S_{i r r}=-\frac{Q_{h}}{T_{h}}+\frac{Q_{c}}{T_{c}}
$$

Equation (80), it should be noted, is what $C T$ considers to be the total or universe entropy change for process (a), i.e.

$$
\Delta S_{C T}[\text { Cycle }]_{i r r}=\Delta S_{\text {rev }}+\Delta S_{i r r}=0+\Delta S_{i r r}=\Delta S_{i r r}
$$

The fact that $\Delta S_{i r}$ is nothing more than the re-expression of Equation (78), i.e. the re-expression of the total entropy change for the irreversible transfer of $Q_{i}$, allows us to relate the previous equation to the lost work associated to this heat transfer, i.e.

$$
\Delta S_{C T}[\text { Cycle }]_{i r r}=\Delta S_{i r r}=\left[Q_{i}\left(T_{h}-T_{c}\right) / T_{h}\right] / T_{c}=\frac{W_{\text {lost }}}{T_{c}}=-\frac{Q_{h}}{T_{h}}+\frac{Q_{c}}{T_{c}}
$$

It is through this connection that we can get a glimpse at the essential message of $C T$. Looking at the previous equation as well as to the representation of the process to which it refers -process 7(c)- we can understand that current second law thermodynamics' considers the 'engine of nature' as having one and only one capability: the wasteful dissipation of gradients i.e. the production of lost work. As can be seen in Equation (81), alien to current second law thermodynamics' entropic balance of the universe is the negentropic contribution associated to the upgrading of heat into work. All the constructive, ordering, and organizing capabilities of work have been erased from thermodynamics by the current formulation. No better explanation of this position can be offered than the desire to retain at all costs the law of increasing entropy as the supreme law of the universe. With unquestionable faith on the dictum Quod non est in formula non est in mondo (von Bertalanffy, 1975, p. 70) the correctors of Clausius' work simply ignored the negentropic contribution of transformation $[Q(T) \rightarrow W]_{r e v}$ and in doing so restricted the domain of application of current second law thermodynamics' to irreversible phenomena. The perceived, actually factual impotence of $C T$ for advancing reasonable, logically sound, and testable models for self-organizing phenomena finds its cause and explanation in the fact that in negating the constructive role of work, $C T$ took away the only analytical tool capable of making sense of these phenomena. The evident contradiction existing between the message of the law of increasing entropy and the order-producing capability of the universe evident within and around us has been succinctly expressed by Caillois as "Clausius and Darwin cannot both be right" (1973). At the eyes of the theories of self-organization finding sustenance in current thermodynamic wisdom, this incessant emergence of order and organization are nothing more than the blind actions of a self-destructing and stupid universe eager to dissolve itself in the Götterdämmerung of its heat death, as according to these theories every self-organized structure takes form and develops at the price of increasing the rate of entropy production (Prigogine, 1980, p. 89)

For the Negentropic Formulation the term $\Delta S_{i r r}$, as given by equation (80), is nothing more than the irreversible component of the total entropy change. For this formulation, the total or universe entropy change is determined, as shown by Equation (77), by both, the reversible and irreversible contributions. The fact that the reversible contribution coming out of process (b) amounts to

$$
\Delta S_{\text {rev }}=-W / T_{h}
$$

Allows us to write as follows the correct expression for the total entropy change of process (a)

$$
\Delta S_{N F}[\text { Cycle }]_{i r r}=-\frac{Q_{h}}{T_{h}}+\frac{Q_{c}}{T_{c}}-\frac{Q}{T_{h}}
$$

Evident in Equation (67) and (84) is the fact that in the $N F$ an equation of the same form applies to reversible and irreversible cyclical processes alike.

\subsection{Irreversible Processes from the Opposite's Perspective}

Let us now agree that as shown in Figure 8, the operation of process (a) is bound by the irreversible and reversible operations shown there, respectively, as (d) and (e). This can be understood by realizing that for the given conditions of operation represented by $Q_{h}$ and the temperatures of the reservoirs, the most efficient operation possible is the reversible operation shown in (e). This operation and its total entropy change $-W_{\text {rev }} / T_{h}$ represent the efficient limit -the limit along a path of increased efficiencies- for process (a) and for its total entropy change. Likewise, the realization that $Q_{h}$ represents the largest amount of heat that can be irreversibly transferred from the hot to the cold reservoir makes of process (d) the most inefficient operation possible. This 
operation and its total entropy change $Q_{h}\left(T_{h}-T_{c}\right) /\left(T_{h} T_{c}\right)$ represent the inefficient limit -the limit along a path of decreasing efficiencies- for process (a) and for its total entropy change. These limiting operations will also be referred to as the reversible and irreversible opposites and respectively symbolized as [REV] and [IRR] .

As it is known, in reversible process $(e)$, where $W=W_{\text {rev }}$, the whole of the universe is susceptible of being restored to its precise initial condition via the simple expedient of feeding $W_{\text {rev }}$ to the reversible inverse of $(e)$. For process $(d)$, where $W=0$, no portion of the universe is susceptible of restoration without the occurrence of additional changes. Here any restoration will come at the price of changes being left in that body called to supply the work required to propel the refrigerator with which the restoration is to be carried through. At the light of the previous considerations it shouldn't be difficult to understand that in process (a), where $0<W<W_{\text {rev }}$, only a portion of the universe can be restored without additional changes setting in. It is in this regard that any such process is partially reversible. This can be understood if it is realized that by feeding the work outputted by process (a) to a reversible refrigerator it is possible to restore to its initial condition that portion of the universe involved in the production of the said work, without the occurrence of any other permanent change. Larger outputs of work lead to larger fractions of the universe susceptible of restoration, and vice versa. Since the whole of the universe is susceptible of being restored when $W=W_{\text {rev }}$, while none is when $W=0$, it follows that the restorable portion i.e. the reversible fraction of the original universe, will be given by $W / W_{\text {rev }}$. This quotient will be subsequently referred to as the reversibility degree, and symbolized as $\phi$, i.e.

$$
\phi=W / W_{\text {rev }}
$$

Obviously, the limit values for $\phi$ are, respectively, zero and one for the irreversible and reversible limits previously described.

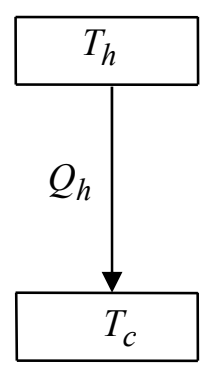

(d)

The irreversible opposite $\eta=0$

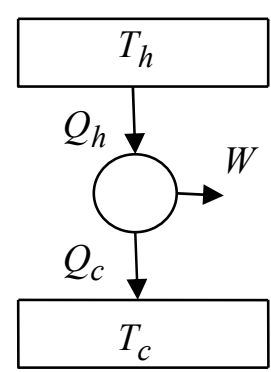

(a)

A simple cyclical process $\eta$

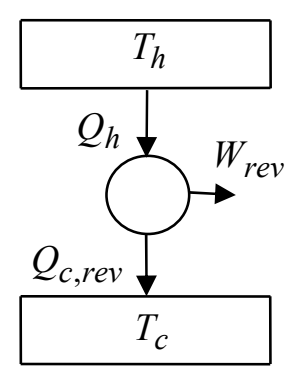

(e)

The reversible
opposite
$\eta_{\text {rev }}$

Figure 8 . The reversible and irreversible opposites to cyclical process (a) are here respectively shown in figures (e) and (d)

It should be noted that an equivalent definition to the one just given can be written for $\phi$ as the quotient of the efficiency of the cyclical process (a) and that of its reversible limit, as follows:

$$
\phi=\eta / \eta_{\text {rev }}
$$

This alternate definition sheds light on the fact that $\phi$ is ultimately a 'normalized' or 'reduced' kind of efficiency.

According to the previous considerations, the total entropy change for process (a) will be limited on the inefficient, irreversible side by the total entropy change of process (d) given below

$$
\left.\Delta S_{N F}[I R R]=\Delta S_{N F}[\phi=0]=Q_{h}\left(T_{h}-T_{c}\right) /\left(T_{h} T_{c}\right)\right\rangle 0
$$

And on the opposite side, the efficient one, the limit will be the negative total entropy change of the reversible limit:

$$
\Delta S_{N F}[R E V]=\Delta S_{N F}[\phi=1]=-\frac{W_{r e v}}{T_{h}}<0
$$


The actual contributions made by these limiting operations to the total entropy change of cyclical process (a) depend on the relative weight each of these extremes has in the actual process. The reversible contribution, determined by the amount of work $W$ actually produced by this cyclical process, will be given by $-W / T_{h}$, which can also be written as $\left(W / W_{\text {rev }}\right)\left(-W_{\text {rev }} / T_{h}\right)$, or as $\phi \Delta S_{N F}[R E V]$. The heat being irreversibly transferred from the hot to the cold reservoir will be quantified as follows: That an amount of work $W$ is produced, with $W\left\langle W_{\text {rev }}\right.$, means that of all the heat given off by the hot reservoir only the portion $\left(Q_{h} / W_{\text {rev }}\right) W$ is used for the production of $W$. The rest: $Q_{h}-\left(Q_{h} / W_{\text {rev }}\right) W$ or $(1-\phi) Q_{h}$ is consequently that being irreversibly transferred; and to this amount corresponds an entropy change of $(1-\phi) Q_{h}\left(T_{h}-T_{c}\right) /\left(T_{h} T_{c}\right)$, which can also be written as $(1-\phi) \Delta S[I R R]$. At the light of the results just given the total entropy change for process (a) can be written as follows:

$$
\Delta S_{N F}[\text { Cycle }]_{i r r}=(1-\phi) \Delta S[I R R]+\phi \Delta S[R E V]
$$

Or as:

$$
\Delta S_{N F}[\text { Cycle }]_{i r r}=\left(1-\frac{W}{W_{\text {rev }}}\right) Q_{h}\left(\frac{T_{h}-T_{c}}{T_{h} T_{c}}\right)-\frac{W}{T_{h}}
$$

The facts that $Q_{h}\left(T_{h}-T_{c}\right) / T_{h}=W_{\text {rev }}$ and $W_{\text {rev }}-W=\left(Q_{h}-Q_{c, \text { rev }}\right)-\left(Q_{h}-Q_{c}\right)=Q_{c}-Q_{c, \text { rev }}$ allows us to write Equation (90) as follows

$$
\Delta S_{N F}[\text { Cycle }]_{i r r}=\frac{Q_{c}}{T_{c}}-\frac{Q_{c, r e v}}{T_{c}}-\frac{W}{T_{h}}
$$

The addition and subtraction of $Q_{h} / T_{h}$ to Equation (91) leads us to

$$
\Delta S_{N F}[\text { Cycle }]_{i r r}=-\frac{Q_{h}}{T_{h}}+\frac{Q_{c}}{T_{c}}-\left(-\frac{Q_{h}}{T_{h}}+\frac{Q_{c, r e v}}{T_{c}}\right)-\frac{W}{T_{h}}
$$

Recognition of the fact that the middle term of the previous equation is equal to zero allows us to write the Negentropic Formulation's expression for the total entropy change of process $(a)$ as follows

$$
\Delta S_{N F}[\text { Cycle }]_{i r r}=-\frac{Q_{h}}{T_{h}}+\frac{Q_{c}}{T_{c}}-\frac{W}{T_{h}}=-\frac{Q_{h}}{T_{h}}+\frac{Q_{c}}{T_{c}}-\frac{Q}{T_{h}}
$$

Beyond the realization that this alternative description of cyclical process $(a)$ leads to the same equation for the total entropy change of an irreversible cyclical process as that obtained in the previous section in reference to Figure 7, it also, and perhaps more important, allows us to realize that the total entropy change of any such process is determined by the weighted contributions of the operations ruling at the opposite ends of the efficiency or reversibility degree domain $[0,1]$. On this perspective the particular entropy change a given cyclical operation may produce will be the result of the particular balance these opposites adopt in the situation being considered. Low values of $\phi$ imply the prevalence of the irreversible opposite and the predominance of its entropic contribution over the negentropic contribution of the reversible opposite. High values of $\phi$ imply, on the other hand, the prevalence of the reversible opposite and the predominance of its negentropic contribution over the entropic contribution of the irreversible opposite. The fact that according to this picture the total entropy change of a cyclical process transits from a positive to a negative value as $\phi$ transits from zero to one, and vice versa, means that there must exist an operation intermediate between the two extremes for which $\Delta S_{N F}[\text { Cycle }]_{i r}=0$. Substitution of this condition in Equation (93) followed by adequately solving for $Q / Q_{h}$, leads to the following expression:

$$
Q / Q_{h}=\frac{T_{h}-T_{c}}{T_{h}+T_{c}}
$$

Replacement of the quotient $Q / Q_{h}$, the efficiency of the operation at the given condition, by $\eta_{u m b r a l}=Q / Q_{h}$, allows us to write Equation (94) as follows

$$
\eta_{\text {umbral }}=\frac{T_{h}-T_{c}}{T_{h}+T_{c}}
$$

The name given to this efficiency comes from the fact that it separates the entropic operations of process (a) from those negentropic, or better said the entropic from the negentropic universes. From our previous definition of $\phi$ it follows that $\phi_{\text {umbral }}=\eta_{\text {umbral }} / \eta_{\text {rev }}$, and if so 


$$
\phi_{\text {umbral }}=\frac{T_{h}}{T_{h}+T_{c}}
$$

In terms of these variables we can say that any operation for which $\phi\left\langle\phi_{\text {umbral }}\right.$ (or $\eta\left\langle\eta_{\text {umbral }}\right.$ ) will take place with a total positive entropy change; while any one for which $\phi\rangle \phi_{\text {umbral }}$ (or $\left.\eta\right\rangle \eta_{\text {umbral }}$ ) with a negative total entropy change. The particular operation for which $\phi=\phi_{\text {umbral }}$ is, on the other hand, non-entropic.

The reader should note that process $7(c)$ is equivalent to the $(1-\phi)$ fraction of process $8(d)$, and process $7(b)$ to the $\phi$ fraction of process $8(e)$

Appropriate combination of Equations (77) and (81) allows us to write the following expression relating the total entropy changes assigned by $N F$ and $C T$ to a Carnot's cycle

$$
\Delta S_{N F}[\text { Cycle }]=\Delta S_{C T}[\text { Cycle }]-(W / T)
$$

From it we learn that the negentropic formulation subsumes current second law thermodynamics' as that special case characterized by $W=0$, or equivalently, by $\eta=0$. In other words it is at the irreversible limit that the negentropic formulation collapses, reduces to $C T$.

\subsection{Back to the Law of Increasing Entropy}

From our previous discussions we have learned that in $N F$, irreversible processes can, depending on their efficiency, take place with positive, negative, or zero total entropy changes, result that denies the notion giving sustenance to the law of increasing entropy, namely, that all irreversible processes take place with positive total entropy changes. This notion is nothing new. It actually originates in Clausius' formulation. To prove this let us recall that in Section 2.12 it was proved, in regard to the irreversible process depicted in Figure 4, that for $T_{h}=600 \mathrm{~K}, T_{c}=400 \mathrm{~K}$, and $Q=1200 \mathrm{~J}$ its total entropy change, as expressed in Equation (36), amounted to $-0.5 \mathrm{JK}^{-1}$, a proof that in Clausius formulation irreversible processes with negative entropy changes are possible. If we repeat these calculations for the irreversible process taking place with $Q=1200 \mathrm{~J}, T_{h}=600 \mathrm{~K}$ and $T_{c}=300 \mathrm{~K}$ we will get a total entropy change in the amount of zero. A final calculation with $Q=1200 \mathrm{~J}$, $T_{h}=600 \mathrm{~K}$ and $T_{c}=298 \mathrm{~K}$ produces for this irreversible process a total entropy change of $0.1 \mathrm{~J} \mathrm{~K}^{-1}$.

The previous argument leads to the inevitable conclusion that the law of increasing entropy, in being incompatible with Clausius' formulation, was dead ab ovo. How then can Clausius' endorsement of such a law be explained? These considerations, in combination with the unexplained shift in values characterizing the transit from Clausius' work to $C T$ evident in Table 2, makes the validity displayed by this law in current second law thermodynamics as if originating in willful design, in decree. (See Note 2)

\subsection{The Negentropic Formulation and Its Testable Prediction}

The conceptual foundation of the negentropic formulation of the second law of thermodynamics is constituted by the non-zero entropy changes assigned in Table 3 to the reversible inter-conversions between heat and work, as well as by its associated concept the W-Transformation. The application of these two notions to the reversible and irreversible operations of Carnot's engine has unveiled two essential facts: (1) that the total entropy change of these processes is determined by the $\phi$-weighted, opposite signs contributions of the reversible and irreversible opposites constituting their efficient and inefficient limits, and (2) the existence of a particular operation -the umbral operation- in which the combination of the said opposite sign contributions, in having the same magnitude, produce a non entropic universe. The relevance of these results consists in the realization that any process working with efficiency smaller than the umbral is entropic, while those with efficiencies larger than the umbral, negentropic. This knowledge is important because it supplies a thermodynamic model for the emergence of order in self-organizing phenomena.

However logical or commonsensical these results appear to be, the fact is that at this point the negentropic formulation is nothing more than the rigorous elaboration of the conjecture assigning non-zero entropy changes to transformations $[Q(T) \rightarrow W]_{r e v}$ and $[W \rightarrow Q(T)]_{r e v}$. In order to become a scientific construction either its basic notions or the results from they obtained must come under the judgment of experience. Let us then propose the following notion as a testable result of the Negentropic Formulation:

The emergence of order in self-organizing phenomena is the tangible, observable manifestation of the transition of the universe of these processes from an entropic to a negentropic condition. In this perspective the so called 'crisis point' at which organization emerges plays a role comparable to the role played by the umbral efficiency in heat engines separating entropic from negentropic operations. 
It needs to be mentioned here that even if the present work is not the first one that this author produce with the non-validity of both, Clausius' and current second law thermodynamics as central theme, it is certainly the last. As such it supersedes all its antecedents, among them (Íñiguez, 1999, 2011, and 2014)

\subsection{A Note on Thomson's Dictum about the Restoration of Mechanical Energy}

In an 1852 paper Lord Kelvin declared "Any restoration of mechanical energy, without more than equivalent of dissipation, is impossible in inanimate material processes, and is probably never effected by means of organized matter, either endowed with vegetable life or subjected to the will of an animated creature." (Thomson, 1852) In order to understand Thomson's dictum as well as to identify the conceptual error behind it let us refer again to Figure 7. As already explained, process 7(a) is a cyclical process of efficiency $\eta$. Its reversible and irreversible components are those sown in Figures 7(b) and 7(c), respectively. As we know, any reversible process produces the maximum possible amount of work at its particular conditions of operation. This means that no work is lost in such a process, or in other words, that the heat discarded to the cold reservoir by any reversible process carries with it no unused or wasted work producing potential. Any claim in the contrary implies the possibility of efficiencies larger than the reversible efficiency. For partially reversible processes the situation is different. Here there is always an amount of heat reaching the cold reservoir having previously bypassed the work producing circuit of the variable body. The amount of work this heat could have produced, but didn't, represent the wasted work producing potential, the lost work. Let us now assume that once a cycle in the operation of 7(a) has taken place, we are to use $W$ to restore to the hot reservoir all of the heat $Q_{c}$ discarded to the cold reservoir. A little pondering about the intended task would convince us that it is impossible. The most $W$ can do, as the inverse of process 7(b) indicates, is to restore $Q_{c}^{*}$ to the hot reservoir. Once this transfer back has been completed, there is still the amount of heat $Q_{i}$ to be transferred but no work left to carry it on. Any attempt to do so requires work to be imported from somewhere else. Under this scheme of things the restoration of $Q_{c}$ not only consumes the work generated in 7(a); it also consumes an extra amount of work for the restoration of $Q_{i}$. At the light of these results Thomson appears to be correct. Any restoration of mechanical energy without further dissipation appears impossible. If the original process had been reversible, $Q_{i}$ would have been zero. In this situation the work produced by 7(a) would have been precisely the amount required to bring $Q_{c}$ back to the hot reservoir. It is only under reversible conditions that on Thomson's perspective we can break even.

This scenario, even if reasonable at first sight, is, however, incorrect. To understand this we have to recall that the amount of heat that in Figure 7 we have represented as $Q_{c}$ subsumes both: $Q_{c}^{*}$ and $Q_{i}$. The fact that the transfer of $Q_{c}^{*}$ is reversible means, as previously noted, that no wasted work producing potential or lost work is carried by it to the cold reservoir. If this is so, then, as a matter of elementary logic, we would have to conclude that if nothing is lost with $Q_{c}^{*}$ then there is nothing from it to be recuperated. If any recuperation, any restoration is to be attempted, it will have to be done in regard to $Q_{i}$ and the unused work producing potential it carries with it in its irreversible flow. The entropy change associated to this irreversible transformation is indicating us that this heat could have delivered an amount of work $Q_{i}\left(T_{h}-T_{c}\right) / T_{h}$. The fact that this is the only mechanical energy lost makes it also the only one that can be recuperated. After all we can't recuperate what we haven't lost, can we? At this point the following question appears natural: at what efficiency should process 7(a) has to operate in order to produce the precise amount of work required for the restoration of $Q_{i}$ through a reversible refrigerator? In order to answer this question let us start writing the following equation for the amount of work required to be outputted by process 7(a) to accomplish this task: $W=Q_{i}\left(T_{h}-T_{c}\right) / T_{c}$. Substitution here of $Q_{i}=Q_{h}-Q_{h}^{*}$ followed by the multiplication of the resulting expression by $\left(T_{c} / T_{h}\right)\left(T_{h} / T_{c}\right)$ leads, after some algebra and further use of the expression $W=Q_{h}^{*}\left(T_{h}-T_{c}\right) / T_{h}$, to the following result: $W=Q_{h}\left(T_{h}-T_{c}\right) /\left(T_{h}+T_{c}\right)$, or equivalently, to the realization that working at the umbral efficiency process 7 (a) outputs the amount of work required for the restoration of $Q_{i}$ to the hot reservoir. Implicit in these results is the notion that in any process working at $\eta>\eta_{\text {umbral }}$ there will be a work surplus after restoration is accomplished. This is the correct perspective to Thomson's dictum (Íñiguez 2012, pp. 97-102).

\subsection{Negentropy as the Arrow of Time}

According to Chaisson (1998, p. 17) "When matter and radiation were still equilibrated in the Radiation Era, only a single temperature is needed to describe the thermal history of the universe...However, once the Matter Era began, matter became atomic, the gas-energy equilibrium was destroyed, and a single temperature was insufficient to specify the bulk evolution of the cosmos. As things turned out, since the random motions of the hydrogen and helium atoms failed to keep pace with the general expansion of the atoms away from one another...the matter cooled faster...than the radiation...Such a thermal gradient is the patent signature of a heat engine, and it is this ever-widening gradient that enabled matter to 'build things'..." 
Once the notions brought forward by the Negentropic Formulation are proved true we will be in the position to assert that this 'building of things' with which Chaisson designates the constructive and organizing activity of the universe, is but the reflection of the fact that this cosmic engine is negentropic. The arrow of time will then take its true form as the indicator of the negentropic evolution of the universe: it will point not toward increasing entropy but toward increasing negentropy.

\section{The Negentropic Formulation at the Light of Related Ideas in Philosophy and Science}

\subsection{Heraclitus and the Logos}

The concept of Opposites in science and philosophy has a long history. It involves Homer, Heraclitus, Nicholas of Cusa, Hegel, Engels, among many others. Even so, Heraclitus appears to be a central reference in this regard. He is usually associated with the phrase panta rhei 'everything flows'. In this regard "His philosophy is seen as an opposite pole from Parmenides, as a dynamic vs. static vision of reality". Central to Heraclitus thought was the notion that behind the omnipresence of change there must exist a rational principle guiding and controlling it i.e. "...the immutable that manifests itself in constant change, the unity that lies at the foundation of the many...". This principle, the Logos, he found on what he called "...the unity of the opposites". Change becomes this way the result, the visible manifestation of "...the tension of the opposites... (of their) strife". It is "Through the constant flow of reality, through the transitions from one opposite to another (that)...regularity, harmony and unity ... transpire..." Taken by themselves "The opposites...lead to disorder. Order is accomplished through the opposites..." (Drozdek, 2007, pp. 27-29). In Heisenberg's opinion “...modern physics is in some way extremely near the doctrines of Heraclitus..." Closer to the problem at hand, he states "The strife between the opposites in the philosophy of Heraclitus can be found in the strife between two different forms of energy" (Heisenberg, 1971, p. 61, 67). In second law thermodynamics, as conceptualized by the Negentropic Formulation, Heisenberg words resonate in the fact that the reversible and irreversible opposites correspond with the predominance of work in the former and heat in the latter.

In consonance with the idea of Marcovich (Drozdek, 2007, footnote p. 28) that "Two opposites form a continuum within every given thing" we have witnessed in our previous discussions how the continuum of different thermodynamic states available to a simple cyclical process subsumes the continuum of different degrees of prevalence of one opposite over the other.

It needs to be noted here that according to the information available to this author, it was Arrhenius whom originally used entropy and negentropy as opposites in thermodynamic arguments. In the words of Kragh: "Arrhenius...objected to Clausius theory of entropy increase, which he found was untenable in its standard formulation, and concluded that on a cosmic scale entropy-increasing processes would be balanced by entropy-decreasing processes... (that) the evolution of the world can continue in an eternal cycle, in which there is neither beginning nor end, and in which life may exist and continue forever and undiminished." (Kragh, 2008, p. 168).

\subsection{Current Views on the Motive Power of the Universe and the Emergence of Life}

In opposition to Thomson's 1852 prediction of the eventual exhaustion of the motive power of the universe, with its concomitant extinction of life and change, there have appeared in recent times a number of notions indicating that not only is the expansion of the universe an inexhaustible source of free energy, but also that it is on reason of the availability of this free energy that life came to be. On these matters Chaisson tells us that "...the necessary (though not necessarily sufficient) condition for the growth of information is guaranteed by the very expansion of the universe. The Universe self-generates a thermal gradient, and increasingly so with time, suggestive of an ever-powerful heat engine were it not for its mechanistic inference. To be sure, we must emphasize throughout the statistical nature of all these processes, meaning that the growth of order is not a foregone conclusion, nor is the Universe a machine. As already noted, thermodynamics tells us if events can occur, not whether they actually will occur. Likewise, this is 'potential' information, realized only should Nature take advantage of the newly established conditions for the development of systems." (Chaisson, 2002, pp. 128-129)

In regard to the question of nature's use of these gradients Morowitz and Smith state that "Life is universally understood to require a source of free energy and mechanisms with which to harness it. Remarkably, the converse may also be true: the continuous generation of sources of free energy by abiotic processes may have forced life into existence as a means to alleviate the buildup of free energy stresses...A deterministic emergence of life would reflect an essential continuity between physics, chemistry, and biology. It would show that a part of the order we recognize as living is thermodynamic order inherent in the geosphere, and that some aspects of 
Darwinian selection are expressions of the likely simpler statistical mechanics of physical and chemical organization." (Morowitz \& Smith, 2006, p. 1)

\subsection{Related Ideas}

The rationalization of life processes in terms of energy forms of higher and lower quality appears to be common theme in biology. On this subject Spier writes: "While flowing through matter, energy inevitably changes from a more to a less productive state. This can be caused by the absorption of some of this energy by the matter that is becoming more complex. Many molecules produced by life, for instance, can only be formed by adding energy. Yet as soon as these forms of greater complexity break down, this energy is released again, although always in a lower-quality form...By flowing through matter, energy always changes from a higher quality to a lower quality form...Apparently, some forms of energy are better able to produce or maintain complexity than others. In the science of thermodynamics the ability of energy to change matter is expressed in the term 'free-energy'." (Spier, 2015, p. 56). The role of work in biological processes can be gauged in the following quote of Ling "A living cell moves, conducts electrical impulses, transports solutes against concentration gradients, and synthesizes chemicals. In these and other activities the cell behaves like a reversible cyclic machine and performs work. Energy is transformed from one form to another in the process." (Ling, 1984, p. 311). The presence of the entropic and negentropic opposites in biological processes can be sensed in the following quote by von Bertalanffy about his 'theory of open systems': "Reduced to a simple and necessarily insufficient formulation, this theory states that growth is the result of a continuous process of build-up and break down in an organism. An organism will grow as long as the anabolic build-up of components exceeds the catabolic break-down; growth comes to a standstill if both processes have reached a steady state." (von Bertalanffy, 1975, p. 47)

It is the belief of the present author that self-organizing processes will be properly understood when the negentropic contribution of energy upgrading processes is properly included in their thermodynamic analysis.

Teilhard de Chardin has precedence in this regard: "Para abarcar completamente la economía evolutiva del universo (incluyendo a la vida), debe añadirse un tercer Principio, el de la Reflexión de la energía, a los ya admitidos, de la conservación y de la degradación de la energía." (De Chardin, 1967, p. 317)

In the present author's translation the previous quote reads as follows "To completely encompass the evolutionary economy of the universe (life included), a third principle must be added, that of the reflection of energy, to those already admitted, about the conservation and degradation of energy." Judging from the context of the quote, the word 'reflection' should be taken as 'upgrading'. After all, what else can you do with energy besides conserving it or degrading it?

\subsection{The Entropy- Negentropy Strife in Fields other than Thermodynamics}

The substitution of Equations (76) and (83) in (77) allows us to write the total entropy change of cyclical processes in the following manner

$$
\Delta S_{N F}[\text { Cycle }]=\frac{W_{\text {lost }}}{T_{c}}-\frac{W_{\text {gained }}}{T_{h}}
$$

In the previous equation $W_{\text {gained }}$ means work gained, this is, the work produced by the cycle.

The very first thing we learn from Equation (98) is that on reason of being $\left.T_{h}\right\rangle T_{c}$, the entropic effect produced by every unit of work lost is larger than the negentropic effect associated to every unit of work gained. If the image is allowed it may very well be said that the penalty for losing work is larger than the reward for gaining it.

Even if a number of vantage points are possible in regard to the interpretation of Equation (98), the discussion will be limited -if the extrapolation is allowed- to the human sphere. Here Equation (98) can be interpreted by saying that the condition of human societies is the resultant of the interplay between two opposing forces: negentropy and entropy, forces which without loss of meaning can be respectively associated with order and chaos and recognized, in the order given, by their respective effects of progress and regress. This perception finds sustenance in the fact that it is $W_{\text {gained }}$ the ordered energy which in combination with the creative power of man's brain and the transforming powers of man's hands has given us knowledge, techniques, and through them the possibility of improving the quality of life for increased numbers of human beings. This is the work that coming out of our energy transforming machines is used for the production of even more efficient energy transforming machines. It has been our increased access to gained work coupled with an increased efficiency in its utilization what has taken human societies through that path recognized as progress, development. This is what has led us to increased amounts and quality of foodstuffs, to schools, research centers, hospitals, vaccines, books, motors, cars, airplanes, boats, tractors, fertilizers, spaceships, the telephone, the computer, etc. In 
summary, it is gained work what propels human societies higher up along the efficiency path, i.e. along the course of progress.

The lost work appearing in Equation (98) includes of course the tax nature imposes on us by way of the always finite difference existing between the efficiency of our energy transforming machines and the reversible efficiency. Beyond this, $W_{\text {lost }}$ finds good exemplification in that portion of $W_{\text {gained }}$ that is generated only to be squandered, misused. We find this wasteful dissipation of our $W_{\text {gained }}$ stock propelling the machinery of consumerism, the same that provides all the gadgetry with which the bored denizens of the so-called industrialized -and not so- nations escape the futility of a life whose success is measured not by the constructive contributions made to society, however modest these contributions might be, but by how much stuff can be bought, or how many material things can be possessed only to be discarded when the late model gadget becomes available. This is also the work used to move the military machinery including that invested in initiating and maintaining wars; but beyond this, it also represents the waste of human lives and human minds through famine, underdevelopment, corruption, illiteracy, disease, discrimination, pollution, drugs and usury; It also takes form in the dilapidation of human potential brought upon by the rampant stupidity of those governments curtailing, inhibiting the development of the creative powers of their population, as well using religion, drugs, race, and similar means to ignite and/or enhance barbaric, animalistic patterns of behavior on their populations; including that malady affecting some so-called emerging societies in which development, understood as increased literacy, better health and human services, extended infrastructure, availability of more and better paid jobs, etc. takes second place to the satisfaction of the extravagant urges and cravings of the political elite constituted in its great majority by semi-literate political gangsters.

It is the relative weight of this lost work in comparison with the previously described gained work what, in the philosophical perspective of Equation (98) separates entropic from negentropic societies. It serves to mention that those societies incapable of recognizing these facts face, inevitably, through the lack of generation or the continued misuse of gained work, an inevitable regress from order to chaos, a situation currently being faced by more than one society on this Earth.

A shorter and definitely more eloquent rendition of the above ideas has been given by Eduardo Césarman, as follows:

"La entropía, como una fuerza prevalente, se hace sentir cuando la violencia triunfa sobre la paz, el odio sobre el amor, la locura sobre la razón, la enfermedad sobre la salud, la miseria sobre la abundancia, la muerte sobre la vida, la ignorancia sobre el conocimiento, la necedad sobre la sabiduría y la mentira sobre la verdad." (Césarman, 1997, p. 17). My English translation reads as follows:

"As a prevalent force entropy makes its presence felt whenever violence triumphs over peace, hate over love, madness over reason, disease over health, poverty over abundance, death over life, ignorance over knowledge, stupidity over wiseness, and falsity over truth."

The results here offered bring forward the realization that in place of the unjustified certainty offered by the law of increasing entropy in the form of the heat death of the universe, a position subsuming the inevitable subjugation of men to forces beyond his control, we find that the fate of our world is determined by the competition of two opposite effects: order-vs.-chaos in the form of negentropy-vs.-entropy, embodied these in the terrestrial, human-centered notions of gained work-vs.-lost work, on whose result the contribution of the human effort is determinant. The future of our Earth and of the human species with it depend no more on the action of metaphysical, eschatological forces; it actually resides in the creative minds and working hands of men.

\subsection{Conclusion}

The evidence advanced in this two-parts work have made clear that the law of increasing entropy is incompatible with the fundamental notions of Clausius' work represented by the values of his transformations. It has also brought to light the fact that the current version of the second law originates in the correction of Clausius' analysis flaws -those made evident in part I of this work- via the arbitrary introduction into the latter of the notion making heat and work energy forms of the same quality in reversible processes. This correction, unknown to most students and studious of thermodynamics, appears to have been performed not with the purpose of unveiling nature's fundamental behavior regarding heat-work inter-conversions, but with the purpose of guaranteeing the absolute validity of the law of increasing entropy. The problem with the correcting notion is that it goes against the recognized intrinsic difference existing between these two energy forms. The arbitrary and unjustified attempt to make identical that which is different is reason enough to reject this correction, and current thermodynamics with it. Coupling this action with the adoption of Clausius' original values for the reversible heat-work inter-conversions constitute the starting point of the revision undertaken by the present author in regard to Clausius' work. Through it 
the up to now unknown notion here called the W-Transformation was unveiled, and with it the correct values for Clausius' transformations emerged. This knowledge is the foundation of the Negentropic Formulation of the second law of thermodynamics. Not only does it unveils the fact that the total entropy change of work producing thermodynamic processes is the resultant of the strife between the entropic -work losing- and negentropic -work gaining- opposites operating at its core. The extrapolation of its conceptual frame to phenomena beyond the thermodynamic domain negates on one side the idea that it is on the action of forces beyond the domain and comprehension of men where the future of the world resides; bringing to life, on the other, the realization that the future of our world, and of our species with it, depends on the actions of men. It is finally up to us whether the world follows the entropic path to self-destruction or the negentropic path of progress, understood, if you allow, as increasing levels of humaneness in mankind.

However plausible, logical, or commonsensical its conceptual frame and the conclusions derived from it might appear, the truth is that the Negentropic Formulation is at this point nothing more than wishful thinking. In order to become a scientific construction or to be rejected for lack of scientific merit it needs to be put under the judgment of experience. With this purpose in mind is that we have advanced the testable notion associating the emergence of order in self-organizing phenomena to the transition of these processes' universe from an entropic to a negentropic condition. In line with Planck's quote stating that "Experiments are the only means of knowledge at our disposal. The rest is poetry, imagination." (wikiquote.org/wiki/Max Planck) we find the present author's belief that the judgment of experience will eventually establish the Negentropic Formulation as the true second law of thermodynamics.

\section{Notes}

1). Further sustenance to this idea is provided by the following material

(a) Those who read the history of scientific advance in terms of an increasingly unbridgeable gulf between scientific and religious metaphysical beliefs must come to terms with the awkwardness of the historical record, which indicates that the scientific, religious and metaphysical beliefs of leading natural scientist interlock and interact, making it quite unrealistic to speak of the 'elimination' of metaphysics from scientific advances. (Mc Grath, 2003, p. 277)

(b) "Two aphorisms have dominated popular discussion of science and religion. The one-Laplace's alleged quip to Napoleon that in his cosmology he had no need of that hypothesis- retains its appeal to those who see in scientific progress a rationale for the exclusion of God-talk. The other-Einstein's formula that science without religion is lame, religion without science blind- has become iconic for those who prefer models of complementarity, even engagement, to those of exclusion....A quite different example, but illustrating the same basic point, would be Fred Hoyle's early preference for a steady-state model of the universe on the ground that it was more congenial to an atheistic worldview than the rival big-bang cosmology" (Osiris, 2001, Science in Theistic Contexts, Vol. 16, pp. vii, viii)

(c) In 1875 the noted British physicist Peter Guthrie Tait (co-author with Lord Kelvin of the book 'Treatise of Natural Philosophy') published, in company of Balfour Stewart, a book titled 'The Unseen Universe' in which they attempted to show that "the presumed incompatibility of Science and Religion does not exist."

These two books are respectively available at https://archive.org/details/treatiseonnatur01darwgoog

book https://archive.org/details/unseenuniverse01unkngoog

(d) Lord Kelvin's “...theological handling of Carnot's law...” in the form of “...religious tirades about 'heat death'..." was criticized by Popper-Lynkeus (1838-1921), a member -along Mach and other noted scientists- of the anti-metaphysical society known as the Vienna Circle. (Byrne et al, 2006, p. 39)

(e) In his 1883 book Dialectics of Nature, Engels forwards the following criticism to Clausius' second law of thermodynamics:

Clausius second law, etc. however it may be formulated, shows energy as lost, qualitatively if not quantitatively. Entropy cannot be destroyed by natural means but it can certainly be created. The world clock has to be wound up, then it goes on running until it arrives at a state of equilibrium from which only a miracle can set it going again. The energy expended in winding has disappeared, at least qualitatively, and can only be restored by an impulse from outside. Hence an impulse from outside was necessary in the beginning also, hence, the quantity of motion, or energy, existing in the universe was not always the same, hence, energy is must have been created, i.e., it must be creatable, and therefore destructible. Ad Absurdum! (Engels, 1964, p. 290) 
It seems pertinent to note that Engels argument just given recreates, in the thermodynamic arena, the Leibniz vs. Clarke (Newton) controversy about 'the best of all possible worlds':

The Newtonians assert that, left to itself, the motive power of the universe would decrease and finally disappear. But, objects Leibniz, if active Force should diminish in the Universe, by the Natural Laws which God has established; so that there should be need for him to give a new Impression in order to restore that Force; like an Artist, Mending the Imperfections of his Machine; the Disorder would not only be with respect to Us, but also with respect to God himself. He might have prevented it and taken better Measures to avoid such an Inconvenience: And therefore, indeed, he has actually done it (Koyre, 2008, p. 145).

(f) In the November 22, 1951 Pope Pius XII Speech to the Pontifical Academy, he stated:

The farther back we go, the more matter presents itself as always more enriched with free-energy, and as a theater of vast cosmic disturbances. Thus everything seems to indicate that the material universe had in finite times a beginning, provided as it was with an indescribably vast abundance of energy reserves, in virtue of which, at first rapidly and then with increased slowness, it evolved into its present state.

[https://www.ewtn.com/library/PAPALDOC/P12EXIST.HTM]

In his November 28, 1951 speech 'Theology and Modern Science' addressed to the Pontifical Academy, Pope Pius XII declared:

Modern science has not only widened and deepened our knowledge of reality and the vastness of the mutability of the cosmos; it likewise provides us with valuable indication on the direction taken by the processes of nature. As late as a hundred years ago, especially after the discovery of the law of the conservation of energy, it was thought that natural processes were reversible. Consequently, in conformity with the principles of strict causality, or rather the determination, of nature, an ever-recurring renovation and rejuvenation of the cosmos was regarded as possible. Through the law of entropy, however, discovered by Rudolf Clausius, it was recognized that the spontaneous processes of nature are always accompanied by a diminution of free and utilizable energy. In a closed material system this conclusion must lead, eventually, to the cessation of processes on a macroscopic scale. This unavoidable fate, from which only hypotheses-sometimes unduly gratuitous-such as that of continued supplementary creation, have endeavored to save the universe, but which instead stands out clearly from positive scientific experience, postulates eloquently the existence of a necessary being.

[http://www.eoht.info/page/Pope+Pius+XII]

2). In regard to the form to be acquired by the final demise predicted by Clausius and $C T$ alike, Kragh tells us that "...according to Thomson it would be a collection of dead stars, (and) according to Clausius a rarefied, uniform gas of particles." Adding, "How seriously can one take a theory that led to such contradictory scenarios?" (Kragh, 2008, p. 45)

It appears appropriate to include here T. S. Elliot's own rendition of the universe's death, as given in the last stanza of his poem 'The Hollow Men': "This is the way the world ends; Not with a bang but a whimper."

Let us finally hear from McGlashan: "The pessimistic idea that the fate of the universe is chaos no doubt has an appeal to a certain kind of mind but it is a matter of faith which has no support from the science of thermodynamics." (1966, p. 232)

Dedication: To my grandsons, Nathan and Noah

\section{References}

Bazarov, I. (1964). Thermodynamics. In Wikiquote. Retrieved from https://en.wikiquote.org/wiki/Thermodynamics

Byrne, J., Toly, N., \& Glover, L. (Eds.). (2009). Transforming Power: Energy, Environment, and Society in Conflict. New Brunswick, N. J.: Transaction Publishers.

Eddington, A. S. (1927). The Nature of the Physical World. New York, The Macmillan Company; Cambridge, Eng., The University Press. Retrieved from https://archive.org/details/natureofphysical00eddi

Caillois, R. (1973). Coherences Aventureuses. Paris: Gallimard.

Sadi-Carnot (2015). Roger Caillois. In eoht. Retrieved from http://www.eoht.info/page/Roger+Caillois

Césarman, E. (1997). Termodinámica de la Vida (Joint publication of the books 'Hombre y Entropía' y 'Orden y Caos'). Editoral Gernika S.A., Mexico, DF.

Chaisson, E. (1998). The Cosmic Environment for the Growth of Complexity, BioSystems 46, pp. 13-19. http://dx.doi.org/10.1016/S0303-2647(97)00076-2 
Chaisson, E. J. (2002). Cosmic Evolution: The Rise of Complexity in Nature. Cambridge USA: Harvard University Press.

Clausius, R. (1879). The Mechanical Theory of Heat. London, UK: Macmillan and Co..

Coveney, P., \& Highfield, R. (1990). The Arrow of Time. New York, NY: Fawcett Columbine.

De Chardin, T. (1976). La Activación de la Energía. Madrid: Taurus Ediciones.

Drozdek, A. (2007). Greek Philosophers as Theologians: The Divine Arche. Burlington VT, USA: Ashgate Publishing Company.

Eddington, A. S. (1929). The Nature of the Physical World. Cambridge, England: The Macmillan Company.

Engels, F. (1964). Dialectics of Nature (3rd Revised ed.). Moscow, USSR: Progress Publishers.

Heisenberg, W. (1958). Physics and Philosophy: The Revolution in Modern Science. London: Unwin University Books, George Allen \& Unwin Ltd.. Retrieved from https://archive.org/details/PhysicsPhilosophy

Íñiguez, J. (1999). A Revision of Clausius work on the Second Law. Entropy, 1, 111-147.

Íñiguez, J. (2011). A Thermodynamic Impasse: A Constant Entropy Irreversible Process. Physical Chemistry: An Indian Journal, 6(3), 125-130.

Íñiguez, J. (2012). A Critical Study of the Law of Increasing Entropy. West Conshohocken, PA: Infinity Publishing Co..

Íñiguez, J. (2014). A Counterexample to the Second Law of Thermodynamics. Applied Physics Research, 6(3), 100-111. http://dx.doi.org/10.5539/apr.v6n3p100

Jantsch, E. (1983). The Self-Organizing Universe. Oxford, UK: Pergamon Press.

Koyre, A. (2008). From the Closed World to the Infinite Universe. Radford VA, USA: A \& D Publishing.

Kragh, H. S. (2008). Entropic Creation: Religious Contexts of Thermodynamics and Cosmology. Burlington VT, USA: Ashgate Publishing Company.

Ling, G. N. (1984). In Search of the Physical Basis of Life. New York, NY: Plenum Press.

McGlashan, M. L. (1966). The Use and Misuse of the Laws of Thermodynamics. J. Chem. Educ., 43(5), 226-232. http://dx.doi.org/10.1021/ed043p226

McGrath, A. E. (2003). A Scientific Theology (Volume 3: Theory). London, UK: T\&T Clark Ltd..

Morowitz, H., \& Smith, E. (2006). Energy Flow and the Organization of Life. SFI working paper 2006-08-029 (pp. 1-9). Retrieved from http://www.santafe.edu/media/workingpapers/06-08-029.pdf

Pitzer, K. S., \& Brewer, L. (1961). Thermodynamics (Revision of Lewis and Randall, International Student Edition). New York, NY: McGraw-Hill.

Prigogine, I. (1980). From Being to Becoming. New York, NY: W. H. Freeman and Company.

Schmidt, E. (1966). Thermodynamics. New York, NY: Dover.

Smith, J. M., \& Van Ness, H. C., Introduction to Chemical Engineering Thermodynamics (2nd ed., International Student Edition). New York, NY: McGraw-Hill.

Spier, F. (2015). Big History and the Future of Humanity. Chichester, West Sussex, UK: John Wiley and Sons.

Thomson, W. (1857). On a Universal Tendency in Nature to the Dissipation of Mechanical Energy. Proceedings of the Royal Society of Edinburgh, 3, 139-142.

von Bertalanffy, L. (1975). Perspectives on General System Theory. New York, NY: George Braziller, Inc..

Webber, H. C., \& Meissner, H. P. (1957). Thermodynamics for Chemical Engineers. New York, NY: John Wiley and Sons.

\section{Copyrights}

Copyright for this article is retained by the author(s), with first publication rights granted to the journal.

This is an open-access article distributed under the terms and conditions of the Creative Commons Attribution license (http://creativecommons.org/licenses/by/3.0/). 